Pacific

Journal of

Mathematics

COMPLETELY REGULAR MULTIVARIATE STATIONARY PROCESSES AND THE MUCKENHOUPT CONDITION

S. Treil and A. Volberg 


\title{
COMPLETELY REGULAR MULTIVARIATE STATIONARY PROCESSES AND THE MUCKENHOUPT CONDITION
}

\author{
S. Treil And A. VolberG
}

\begin{abstract}
We are going to give necessary and sufficient conditions for a multivariate stationary stochastic process to be completely regular. We also give the answer to a question of V.V. Peller concerning the spectral measure characterization of such processes.
\end{abstract}

\section{Introduction.}

In this paper we shall give a necessary and sufficient condition for a multivariate stationary stochastic process to be completely regular. For the scalar case the description of completely regular processes was obtained by Helson an Sarason, see $[\mathbf{2}, \mathbf{9}]$. Almost none of the scalar methods is available in the vector situation. The explanation is simple. Our problem will be reduced to verifying $L^{2}$ weighted inequalities for a certain integral operator. The weight will be a matrix weight arising from the spectral measure of the process. All the pointwise estimates of integral operators become too crude for the vector valued case. For example, if a positive kernel is majorized by another one, and this second kernel gives the bounded operator in $L^{2}(\mu)$, then the original kernel obviously corresponds to a bounded operator in $L^{2}(\mu)$ too. But this is not the case if $\mu$ is a matrix measure even for scalar kernels.

The study of prediction theory for multivariate stationary stochastic processes was started by Kolmogorov and Wiener in the 50's, see, for example [13], [14], and [4]. It was later continued in works of I. Ibragimov, Yu. Rozanov, V. Solev, A. Yaglom, V. Peller, S. Khruschev, N.J. Young. An extensive bibliography can be found in [6] (for scalar processes) and in [5] (for vector ones).

Let us recall that a multivariate stationary stochastic process with discrete time is a sequence of $d$-tuples $x(n)=\left(x_{1}(n), x_{2}(n), \ldots, x_{d}(n)\right), n \in \mathbb{Z}$ of scalar random variables such that $\mathbb{E}\left|x_{j}(n)\right|^{2}<\infty$ and the correlation matrix $Q(n, k)$

$$
Q(n, k)=\left\{Q(n, k)_{i, j}\right\}_{1 \leq i, j \leq d}:=\left\{\mathbb{E} x_{i}(n) \overline{x_{j}(k)}\right\}_{1 \leq i, j \leq d}
$$

depends only on the difference $n-k$; here $\mathbb{E}$ denotes mathematical expectation. 
It is well known (see [8]) that there exists a matrix-valued nonnegative measure $M$ on the unit circle $\mathbb{T}$ whose Fourier coefficients coincide with entries of the correlation matrix

$$
Q(n, k)=\widehat{M}(n-k), \quad n, k \in \mathbb{Z} .
$$

The measure $M$ is called the spectral measure of the process $\{x(n)\}_{n \in \mathbb{Z}}$.

The random variables $x_{j}(n)$ can be treated as elements of Hilbert space $L^{2}(\Omega, d P)$, where $\Omega$ is the probability space and $P$ is the probability, so $x(n)$ can be treated as elements of the $\mathbb{R}^{d}$-valued $L^{2}$ space $L_{\mathbb{R}^{d}}^{2}(\Omega, d P)$ For a moment $n$ of time we can consider the past $\mathcal{X}_{n}$ and the future $\mathcal{X}^{n}$ of the process, which are defined as the subspaces

$$
\begin{aligned}
& \mathcal{X}_{n}=\operatorname{span}\left\{x_{j}(k): 1 \leq j \leq d, k<n\right\} \\
& \mathcal{X}^{n}=\operatorname{span}\left\{x_{j}(k): 1 \leq j \leq d, k \geq n\right\}
\end{aligned}
$$

of $L^{2}(\Omega, d P)$.

A process is called regular if $\cap_{n \geq 0} \mathcal{X}^{n}=\{0\}$. In this case (see [8]) the spectral measure $M$ of the process is absolutely continuous with respect to Lebesgue measure. Let $W$ be the density of $M$ with respect to Lebesgue measure. The matrix-valued function $W$ is called the spectral density of the process.

A process $\{x(n)\}_{n \in \mathbb{Z}}$ is called completely regular if its past is asymptotically orthogonal to the future, namely if

$$
\sup \left\{|\mathbb{E}(\xi \eta)|: \xi \in \mathcal{X}_{0}, \eta \in \mathcal{X}^{n}, \mathbb{E}|\xi|^{2} \leq 1, \mathbb{E}|\eta|^{2} \leq 1\right\} \longrightarrow 0 \quad \text { as } n \rightarrow \infty \text {. }
$$

Of course, complete regularity implies regularity. If the process is Gaussian (i.e. all random variables $x_{j}(k)$ have normal distribution) then the complete regularity means simply that past and future are almost independent. The problem we are dealing with is to characterize completely regular processes in terms of spectral measure.

It has been already mentioned (see again [8]) that if the process is completely regular, then its spectral measure is absolutely continuous, $d M=$ $W d m$ where $d m$ is the normalized $(m(\mathbb{T})=1)$ Lebesgue measure on the unit circle $\mathbb{T}$.

The reader is referred to $[\mathbf{8}]$ once more to see that there exists $d_{0} \leq d$ (the rank of the process) such that the spectral density $W(t)$ has rank $d_{0}$ for almost all $t \in \mathbb{T}$. If $d_{0}=d$ then the process $\{x(n)\}$ is said to be a full rank.

The study of processes of arbitrary rank can be easily reduced to the study of the processes of full rank, see [3]. So in this paper we shall consider only processes of full rank.

For the scalar case the description of completely regular processes was obtained by Helson an Sarason, see [2, 9]. To state their result we need a couple of definitions. 
Let us recall that a function $f$ on the unit circle $\mathbb{T}$ belongs to the space BMO (bounded mean oscillation) if

$$
\sup _{I} \frac{1}{|I|} \int_{I}\left|f-f_{I}\right| d m=\|f\|_{\mathrm{BMO}}<\infty ;
$$

here $f_{I}$ denotes the mean value of $f$ on the interval $I: f_{I}:=|I|^{-1} \int_{I} f d m$ and the supremum is taken over all subarcs $I$ of $\mathbb{T}$.

The space VMO (vanishing mean oscillation) consists of all function $f \in$ BMO such that

$$
\sup _{I} \frac{1}{|I|} \int_{I}\left|f-f_{I}\right| d m \longrightarrow 0 \quad \text { as }|I| \rightarrow 0 .
$$

Theorem 1.1 (Helson, Sarason). Let $w$ be the spectral density of a scalar stationary process. Then the process is completely regular if and only if $w$ admits a representation

$$
w=|p|^{2} e^{\varphi},
$$

where $p$ is a polynomial with roots on the unit circle $\mathbb{T}$ and $\varphi$ is a real-valued function in VMO.

It was conjectured by V. Peller in [5] that the same result holds for multivariate stationary processes. Namely he conjectured that a multivariate stationary process is completely regular if and only if its spectral density $W$ admits the following representation

$$
W=P^{*} e^{\Phi} P,
$$

where $P$ is a polynomial matrix whose determinant has roots on $\mathbb{T}$ and the matrix function $\Phi=\Phi^{*}$ belongs VMO.

In this direction he was able to prove the following theorem:

Theorem 1.2. A multivariate stationary process is completely regular if and only if its spectral density $W$ admits the factorization

$$
W=P^{*} W_{1} P,
$$

where $P$ is a polynomial matrix whose determinant has roots on $\mathbb{T}$ and $W_{1}$ is the density of a completely regular stationary process such that $W_{1}^{-1} \in L^{1}$.

1.1. The main result. Let us recall that a measure $\mu$ on the unit disk $\mathbb{D}$ is called Carleson if

$$
\sup _{I} \mu(Q(I)) \leq C \cdot|I|
$$

and is called the vanishing Carleson measure if

$$
\limsup _{|I| \rightarrow 0} \mu(Q(I)) /|I|=0
$$

where limsup is taken over all subarcs $I$ of $T$. Here $Q(I)$ denotes the "Carleson square" for the arc $I$,

$$
Q(I)=\{z \in \mathbb{D}: z /|z| \in I, 1-|I| \leq|z|<1\} .
$$


For a function $F$ on the unit circle let $F(\lambda), \lambda \in \mathbb{D}$, denote its harmonic extension at the point $\lambda$.

The main result of the paper is the following theorem.

Theorem 1.3. Let the density $W$ of a stationary process satisfy $W^{-1} \in L^{1}$. Then the the following are equivalent:

1) The process is completely regular;

2) $W^{-1}$ is the spectral density of a completely regular process;

3) $\limsup _{|I| \rightarrow 0}\left\|\left(\frac{1}{|I|} \int_{I} W d m\right)^{1 / 2}\left(\frac{1}{|I|} \int_{I} W^{-1} d m\right)^{1 / 2}\right\|=1$; here supremum is taken over all subarcs $I$ of $\mathbb{T}$;

4) $\limsup _{|\lambda| \rightarrow 1}\left\|(W(\lambda))^{1 / 2}\left(W^{-1}(\lambda)\right)^{1 / 2}\right\|=1$, where $W(\lambda)$ and $W^{-1}(\lambda)$ are harmonic extensions of functions $W \mid \mathbb{T}$ and $W^{-1} \mid \mathbb{T}$ respectively at point $\lambda \in \mathbb{D}$.

5) $\limsup _{|\lambda| \rightarrow 1}\{\operatorname{det}(W(\lambda)) \exp (-[\log \operatorname{det} W](\lambda))\}=1$, where $W(\lambda)$ and $[\log \operatorname{det} W](\lambda)$ are harmonic extensions of functions $W \mid \mathbb{T}$ and $\log \operatorname{det} W \mid \mathbb{T}$ respectively at point $\lambda \in \mathbb{D}$.

6) The measures

$$
\left\|W(z)^{-1 / 2}\left(\frac{\partial}{\partial x} W(z)\right) W(z)^{-1 / 2}\right\|^{2}\left(1-|z|^{2}\right) d x d y
$$

and

$$
\left\|W(z)^{-1 / 2}\left(\frac{\partial}{\partial y} W(z)\right) W(z)^{-1 / 2}\right\|^{2}\left(1-|z|^{2}\right) d x d y
$$

are vanishing Carleson measures.

Together with Theorem 1.2 the above theorem yields the complete description of completely regular stationary processes.

Theorem 1.4. A stationary process with spectral density $\mathcal{W}$ is completely regular if and only if $\mathcal{W}$ admits the representation

$$
\mathcal{W}=P^{*} W P,
$$

where $P$ is a polynomial matrix whose determinant has roots on $\mathbb{T}$ and the matrix-function $W$ satisfies $W^{-1} \in L^{1}$ and one of equivalent conditions 3-6 of Theorem 1.3.

Let us discuss the main result (Theorem 1.3) a little bit. First of all it is not difficult to show directly that in the scalar case the conditions 3-6 of Theorem 1.3 are equivalent to $W=e^{\varphi}, \varphi \in \mathrm{VMO}$. We are leaving this as an exercise for the reader. 
Usually in probability only real valued stationary processes are considered. In that case the spectral density of a process should satisfy $W(\bar{z})=W(z)$, and only such functions can be realized as densities of stationary processes.

If one allow complex-valued processes, any nonnegative matrix function is the spectral density of some stationary process.

Our theorem deals with arbitrary nonnegative matrix-functions and can be applied to complex-valued processes (as well as to real-valued).

\section{Scheme of the proof of the main result.}

The diagram of the proof will be the following: $1 \Longrightarrow 4 \Longrightarrow 5 \Longrightarrow 6 \Longrightarrow 1$. Then we will show that $1 \Longrightarrow 2$ and so automatically $2 \Longrightarrow 1$.

And in this section we will show that $3 \Longleftrightarrow 4$.

Lemma 2.1. For a scalar weight $w$ the following conditions are equivalent:

1) $\limsup _{|I| \rightarrow 0}\left(\frac{1}{|I|} \int_{I} w\right)\left(\frac{1}{|I|} \int_{I} w^{-1}\right)=1$;

2) $\lim \sup w(\lambda) w^{-1}(\lambda)=1$, where $w(\lambda)$ and $w^{-1}(\lambda)$ denote the harmonic $|\lambda| \rightarrow 1$

extensions of $w$ and $w^{-1}$ respectively at the point $\lambda$;

3) $w=e^{\varphi}$, where $\varphi \in V M O$.

Proof. First of all let us rewrite condition 1. Let $\varphi:=\log w$. For a function $f$ let $f_{I}$ denote its average over the $\operatorname{arc} I, f_{I}:=|I|^{-1} \int_{I} f$. Then clearly

$$
w_{I} \cdot\left(w^{-1}\right)_{I}=\left[w_{I} \exp \left(-\varphi_{I}\right)\right] \cdot\left[\left(w^{-1}\right)_{I} \exp \left(\varphi_{I}\right)\right] .
$$

By Jensen inequality (geometric mean $\leq$ arithmetic mean) the expressions in brackets are at least 1 , so the condition 1 splits into the following 2 conditions

$$
\limsup _{|I| \rightarrow 0}\left[w_{I} \exp \left(-\varphi_{I}\right)\right]=1, \quad \text { and } \quad \limsup _{|I| \rightarrow 0}\left[\left(w^{-1}\right)_{I} \exp \left(\varphi_{I}\right)\right]=1 .
$$

Let $f_{+}$denote the positive part of the function $f, f_{+}(x):=\max (f(x), 0)$. Then the inequality

$$
x \leq e^{x}-1 \quad \text { for } x \geq 0
$$

implies

$$
\begin{aligned}
\frac{1}{|I|} \int_{I}\left(\varphi-\varphi_{I}\right)_{+} & \leq \frac{1}{|I|} \int_{I}\left(\exp \left(\varphi-\varphi_{I}\right)-1\right) \\
& =w_{I} \exp \left(-\varphi_{I}\right)-1 \rightarrow 0 \quad \text { as }|I| \rightarrow 0 .
\end{aligned}
$$

Since $\int_{I}\left|\varphi-\varphi_{I}\right|=2 \int_{I}\left(f-f_{I}\right)_{+}$, one can conclude that $\varphi \in$ VMO.

Similarly, using Poisson averages instead of averages over intervals one can get from condition 2 of the lemma that harmonic extension of $|\varphi-\varphi(\lambda)|$ at the point $\lambda$ tends to 0 as $\lambda \rightarrow 1$. But that is an equivalent definition of $\mathrm{VMO}$, so the condition 2 also implies that $\varphi \in \mathrm{VMO}$. 
On the other hand, if $\varphi \in \mathrm{VMO}$, John-Nirenberg Theorem (see [1, Chapter VI]) claims that the measure of the set $\left\{t \in I:\left|\varphi(t)-\varphi_{I}\right|>a\right\}$ is estimated from above by $C e^{-K a}$, where $K=K_{I} \rightarrow \infty$ as $|I| \rightarrow 0$. Therefore for $x>1$ the measure of the set $\left\{t \in I: \exp \left(\varphi(t)-\varphi_{I}\right)>x\right\}$ is estimated from above by $C x^{-K}$. Integrating this distribution function one can get that $\lim \sup _{|I| \rightarrow 0} w_{I} \exp \left(-\varphi_{I}\right) \leq 1$ (in fact, it is 1 , because by Jensen inequality $\left.w_{I} \exp \left(-\varphi_{I}\right) \geq 1\right)$. Similarly, $\lim \sup _{|I| \rightarrow 0}\left(w^{-1}\right)_{I} \exp \left(\varphi_{I}\right)=1$. Multiplying the above two inequalities one gets condition 1 .

The proof that $3 \Longrightarrow 2$ is similar. For a point $\lambda \in \mathbb{D}$ let $I_{\lambda}$ be an interval with center at $\lambda /|\lambda|$ of length $(1-|\lambda|)^{1 / 3}$. Since the Poisson Kernel $P_{\lambda}(z)=\left(1-|\lambda|^{2}\right) \cdot|1-\bar{\lambda} z|^{-2}$ satisfies $\sup _{z \in \mathbb{T} \backslash I_{\lambda}} P_{\lambda}(z) \rightarrow 0$ as $|\lambda| \rightarrow 1$, the distribution inequality for $\varphi$ on $I_{\lambda}$ implies that $w(\lambda) \cdot \exp (-\varphi(\lambda)) \rightarrow 1$ as $|\lambda| \rightarrow 1$, and therefore the condition 2 of the lemma.

The following Lemma is probably well known and can be easily from the distribution function inequality for VMO (John-Nirenberg Theorem).

Lemma 2.2. For $\lambda \in \mathbb{D}$ let $I_{\lambda}$ be an interval centered at $\lambda /|\lambda|$ of length $1-|\lambda|$. If $\varphi \in V M O$, then $\varphi_{I_{\lambda}}-\varphi(\lambda) \rightarrow 0$ as $|\lambda| \rightarrow 1$.

Corollary 2.3. Let $\varphi \in V M O$ and let $w=e^{\varphi}$. Then for $I_{\lambda}$ as in the above lemma we have

$$
\lim _{|\lambda| \rightarrow 1} \frac{w(\lambda)}{w_{I_{\lambda}}}=1 .
$$

Proof. By the above lemma $\lim _{|\lambda| \rightarrow 1} \exp (\varphi(\lambda)) / \exp \left(\varphi_{I_{\lambda}}\right)=1$. On the other hand it follows from the proof of Lemma 2.1 that

$$
\lim _{|\lambda| \rightarrow 1} w(\lambda) / \exp (\varphi(\lambda))=1 \quad \text { and } \quad \lim _{|I| \rightarrow 0} w_{I} / \exp \left(\varphi_{I}\right)=1 .
$$

Taking the ration of the last 2 identities (with $I=I_{\lambda}$ ) we get the statement we need.

Now to show equivalence of condition 3 and 4 of Theorem 1.3 is enough to show that these conditions imply that for a fixed vector $e \in \mathbb{C}^{d}$ scalar weight $w(z)=(W(z) e, e)$ satisfies conditions 1 and 2 of Lemma 2.1. Then Corollary 2.3 implies that the averages $W_{I_{\lambda}}$ and $W(\lambda)$ are equivalent, the same holds for $W^{-1}$, and we are done.

It remains now to show that the scalar weight $w(z)=(W(z) e, e)$ satisfies condition 1 (equivalently 2) of Lemma 2.1. The easiest way to do that is to recall where the Muckenhoupt condition $\left(A_{2}\right)$ came from, see [10].

Recall that the quantity $\left\|\left[W_{I}\right]^{1 / 2}\left[\left(W^{-1}\right)_{I}\right]^{1 / 2}\right\|$ is just the norm of the averaging operator $f \mapsto f_{I} \cdot \chi_{I}$ in the weighted space $L^{2}(W)$, see $[\mathbf{1 0}$, Lemma 2.1]. Then $\left[w_{I}\right]^{1 / 2}\left[\left(w^{-1}\right)_{I}\right]^{1 / 2}$ is the norm of the restriction of the above 
averaging operator onto the subspace of $L^{2}(W)$ consisting of functions of form $f e$ where $f$ is a scalar function. Therefore

$$
1 \leq\left[w_{I}\right]^{1 / 2}\left[\left(w^{-1}\right)_{I}\right]^{1 / 2} \leq\left\|\left[W_{I}\right]^{1 / 2}\left[\left(W^{-1}\right)_{I}\right]^{1 / 2}\right\|
$$

so the weight $w$ satisfies condition 1 of the lemma.

Similarly, the quantity $\left\|W(\lambda)^{1 / 2} W^{-1}(\lambda)^{1 / 2}\right\|$ is just the norm of another averaging operator $\left(f \mapsto \int_{\mathbb{T}} f k_{\lambda}\right) k_{\lambda}$, where $k_{\lambda}$ is the normalized reproducing kernel of $H^{2}, k_{\lambda}(z)=\left(1-|\lambda|^{2}\right)^{1 / 2}(1-\bar{\lambda} z)^{-1}$, see [10, Lemma 2.1], so condition 4 of the theorem implies condition 2 of the lemma for the weight $w$.

\section{Eliminating probability.}

The problem of description of completely regular processes can be now stated without mentioning any probability theory at all.

First of all notice that without loss of generality we can assume that the process is complex-valued. Namely, if we have a real stationary process $\{x(n)\}_{n \in \mathbb{Z}}$ we can consider its comlexification, namely the same process but in the complex Hilbert space $L_{\mathbb{C}^{d}}^{2}(\Omega, d P)$. Consider the comlexificated past $\left(\mathcal{X}_{n}\right)_{\mathbb{C}}$ and future $\left(\mathcal{X}^{n}\right)_{\mathbb{C}}$

$$
\begin{aligned}
\left(\mathcal{X}_{n}\right)_{\mathbb{C}} & =\operatorname{span}\left\{x_{j}(k): 1 \leq j \leq d, k<n\right\} \\
\left(\mathcal{X}^{n}\right)_{\mathbb{C}} & =\operatorname{span}\left\{x_{j}(k): 1 \leq j \leq d, k \geq n\right\}
\end{aligned}
$$

where span now means the closed linear span in the complex Hilbert space $L_{\mathbb{C}^{d}}^{2}(\Omega, d P)$. It is easy to see that

$$
\begin{aligned}
& \sup \left\{|\mathbb{E}(\xi \eta)|: \xi \in \mathcal{X}_{0}, \eta \in \mathcal{X}^{n}, \mathbb{E}|\xi|^{2} \leq 1, \mathbb{E}|\eta|^{2} \leq 1\right\} \\
& \quad=\sup \left\{|\mathbb{E}(\xi \bar{\eta})|: \xi \in\left(\mathcal{X}_{0}\right)_{\mathbb{C}}, \eta \in\left(\mathcal{X}^{n}\right)_{\mathbb{C}}, \mathbb{E}|\xi|^{2} \leq 1, \mathbb{E}|\eta|^{2} \leq 1\right\},
\end{aligned}
$$

so a process and its comlexification are completely regular simultaneously. So we indeed can assume from the beginning that our process is complex valued.

Consider now the vector space $L^{2}(W)$ of $\mathbb{C}^{d}$-valued functions on the unit circle with the norm

$$
\|f\|_{L^{2}(W)}^{2}=\int_{\mathbb{T}}(W(\xi) f(\xi), f(\xi))_{\mathbb{C}^{d}} d m(\xi)
$$

(of course we have to take the quotient space over the functions of norm $0)$. The mapping $x_{j}(k) \mapsto z^{k} e_{j}$, where $e_{j}, j=1, \ldots, d$ is the standard orthonormal basis in $\mathbb{C}^{d}$, is an isometric isomorphism between $\operatorname{span}\left\{x_{j}(k)\right.$ : $1 \leq j \leq d, k \in \mathbb{Z}\}$ and $L^{2}(W)$. 
The past $\mathcal{X}_{n}$ and future $\mathcal{X}^{n}$ are mapped to the spaces $X_{n}$ and $X^{n}$ of $L^{2}(W)$

$$
\begin{aligned}
& X_{n}=\operatorname{span}\left\{z^{k} \mathbb{C}^{d}: k<n\right\} \\
& X^{n}=\operatorname{span}\left\{z^{k} \mathbb{C}^{d}: k \geq n\right\} .
\end{aligned}
$$

So the problem of describing completely regular stationary processes can be reformulated as follows: Describe all matrix weights $W$ such that the spaces $X_{0}$ and $X^{n}$ are asymptotically $($ as $n \rightarrow \infty)$ orthogonal to each other,

$$
\begin{gathered}
\rho_{n}=\sup \left\{\left|(\xi, \eta)_{L^{2}(W)}\right|: \xi \in X_{0}, \eta \in X^{n}\right. \\
\left.\|\xi\|_{L^{2}(W)} \leq 1,\|\eta\|_{L^{2}(W)} \leq 1\right\} \longrightarrow 0,
\end{gathered}
$$

as $n \rightarrow \infty$.

\section{Necessity $(1 \Longrightarrow 4)$.}

In this section we are going to prove the implication $1 \Longrightarrow 4$ (see Theorem 4.1 below) and the equivalence $1 \Longleftrightarrow 2$ (see Lemma 4.4).

For a function $F$ defined on the unit circle $\mathbb{T}$ let $F(\lambda)$ denote its harmonic extension at the point $\lambda \in \mathbb{D}$.

Theorem 4.1. Let $W$ be a matrix valued weight such that $W^{-1} \in L^{1}$. Suppose the "past" $X_{0}$ and "future" $X^{n}$ defined by (3.1), (3.2) are asymptotically orthogonal, which is

$$
\begin{aligned}
& \rho_{n}=\sup \left\{\left|(\xi, \eta)_{L^{2}(W)}\right|: \xi \in X_{0}, \eta \in X^{n}\right. \\
&\left.\|\xi\|_{L^{2}(W)} \leq 1,\|\eta\|_{L^{2}(W)} \leq 1\right\} \longrightarrow 0
\end{aligned}
$$

as $n \rightarrow \infty$. Then

$$
\limsup _{|\lambda| \rightarrow 1}\left\|(W(\lambda))^{1 / 2}\left(W^{-1}(\lambda)\right)^{1 / 2}\right\|=1 .
$$

Proof. First of all let us show that if $W^{-1}$ is completely regular and $W^{-1} \in$ $L^{1}$ then $W$ satisfies the Muckenhoupt $\left(A_{2}\right)$ condition

$$
\sup _{\lambda \in \mathbb{D}}\left\|(W(\lambda))^{1 / 2}\left(W^{-1}(\lambda)\right)^{1 / 2}\right\|<\infty .
$$

Recall that $\left\|(W(\lambda))^{1 / 2}\left(W^{-1}(\lambda)\right)^{1 / 2}\right\|$ is exactly the norm of the operator $f \mapsto\left(f, k_{\lambda}\right) k_{\lambda}$ in the weighted space $L^{2}(W)$; here $k_{\lambda}$ denotes the normalized reproducing kernel for $H^{2}$,

$$
k_{\lambda}(z):=\frac{\left(1-|\lambda|^{2}\right)^{1 / 2}}{1-\bar{\lambda} z}, \quad \lambda \in \mathbb{D},
$$


$\left\|k_{\lambda}\right\|_{2}=1$. Note that $k_{0} \equiv 1$. So if $W^{-1} \in L^{1}$ the operator $f \mapsto(f, 1) 1$ is bounded in $L^{2}(W)$, and therefore by translation invariance the operators $f \mapsto\left(f, z^{n}\right) z^{n}=\hat{f}(n) z^{n}$ are bounded as well (they all have the same norm).

We know that the spaces $X_{0}$ and $X^{n}$ are asymptotically orthogonal, so we can say that for large enough $N$ the operator $P_{+}$restricted onto $\operatorname{span}\left\{X_{0}, X^{N}\right\}=\operatorname{span}\left\{z^{n} \mathbb{C}^{d}: n \notin[0, N]\right\}$ is bounded, say by 2 ,

$$
\left\|P_{+} f\right\|_{L^{2}(W)} \leq 2\|f\|_{L^{2}(W)}, \forall f \in \operatorname{span}\left\{X_{0}, X^{N}\right\}=\operatorname{span}\left\{z^{n} \mathbb{C}^{d}: n \notin[0, N]\right\} .
$$

Since $f-\sum_{n=0}^{N} \hat{f}(n) z^{n} \in \operatorname{span}\left\{X_{0}, X^{N}\right\}=\operatorname{span}\left\{z^{n} \mathbb{C}^{d}: n \notin[0, N]\right\}$, one can conclude that the operator $P_{+}$is bounded in $L^{2}(W)$, and so the weight satisfies the Muckenhoupt condition $\left(A_{2}\right)$.

We will need the following simple lemma about Muckenhoupt weights.

Lemma 4.2. If $w$ is a scalar Muckenhoupt weight, then its harmonic extension $w(\lambda)$ cannot decay too fast near the boundary of the disk. Namely, if the Muckenhoupt norm of $w$ is at most $M$ there is a function $\alpha=\alpha_{M}$, $\alpha:[0,1) \rightarrow(0, \infty), \alpha(t) \searrow 0$ as $t \rightarrow 1+$ such that

$$
\frac{\left(1-|\lambda|^{2}\right) w(0)}{w(\lambda)} \leq \alpha(|\lambda|) .
$$

Proof of the lemma. For an arc $I \subset \mathbb{T}$ and $k>0$ let $k I$ denote the arc of length $k|I|$ with the same center as $I$.

We are going to show that for a Muckenhoupt weight $w$ with the Muckenhoupt norm at most $M$

$$
w_{2^{n} I} \leq M^{2}(2-\varepsilon)^{n} w_{I}, \quad \varepsilon=\varepsilon(M)>0 .
$$

Applying this formula in the case $2^{n} I=\mathbb{T}$ and using the trivial estimate

$$
w(\lambda) \geq C w_{I_{\lambda}}
$$

where $I_{\lambda}$ is the arc with center at the point $\lambda /|\lambda|,\left|I_{\lambda}\right|=1-|\lambda|^{2}$ and $C$ is an absolute constant, we can get from there (recall that $\left|I_{\lambda}\right|=1-|\lambda|^{2}=2^{-n}$ ) $w(\lambda) \geq c(2-\varepsilon)^{-n} \cdot w(0)=c(2-\varepsilon)^{\log _{2}\left(1-|\lambda|^{2}\right)} \cdot w(0)=c \cdot(e-\delta)^{\log \left(1-|\lambda|^{2}\right)} \cdot w(0)$, where $\delta=\delta(\varepsilon)>0$; here $e$ is the base of the natural logarithm, not a vector in $\mathbb{C}^{d}$. This estimate implies the conclusion of the lemma with $\alpha(t)=$ $c^{-1}\left(1-t^{2}\right) \cdot(e-\delta)^{-\log \left(1-t^{2}\right)}$.

To prove (4.1) we notice the since the weight $w^{-1}$ is the Muckenhoupt $\left(A_{2}\right)$ weight with the same Muckenhoupt norm as $w$, it is doubling and therefore

$$
\left(w^{-1}\right)_{2 I} \geq(2-\varepsilon)^{-1}\left(w^{-1}\right)_{I}
$$

where $\varepsilon$ depends only on the Muckenhoupt norm of $w$. Iterating this inequality $n$ times we get

$$
\left(w^{-1}\right)_{2^{n} I} \geq(2-\varepsilon)^{-n}\left(w^{-1}\right)_{I} .
$$


The last estimate and the Muckenhoupt condition imply

$$
w_{2^{n} I} \leq M /\left(w^{-1}\right)_{2^{n} I} \leq M \cdot(2-\varepsilon)^{n} /\left(w^{-1}\right)_{I} \leq M^{2}(2-\varepsilon)^{n} w_{I}
$$

and that is exactly what we need.

Corollary 4.3. If a matrix weight $W$ satisfies the Muckenhoupt condition $\left(A_{2}\right)$ with the Muckenhoupt norm at most $M$ then for any $e \in \mathbb{C}^{d}$

$$
\left(1-|\lambda|^{2}\right) \cdot \frac{(W(0) e, e)_{\mathbb{C}^{d}}}{(W(\lambda) e, e)_{\mathbb{C}^{d}}} \leq \alpha(|\lambda|) \rightarrow 0 \quad \text { as }|\lambda| \rightarrow 1,
$$

where $\alpha=\alpha_{M}$ is the function from Lemma 4.2 .

Proof of the corollary. The proof follows immediately from the fact that the scalar weight $w, w(\xi)=(W(\xi) e, e)_{\mathbb{C}^{d}}$ is the Muckenhoupt $\left(A_{2}\right)$ weight with the Muckenhoupt norm at most $M$ (see [11], proof of Corollary 2.4).

We now return to the proof of the theorem.

The condition $W^{-1} \in L^{1}$ implies that $\int_{\mathbb{T}} \log \operatorname{det} W(\xi) d m(\xi)>-\infty$, hence (see [7]) there exists a factorization of $W$ of the form $W=F^{*} F$, where $F$ is an outer matrix function in $H^{2}$.

Take $e \in \mathbb{C}^{d}$ and let us compute the distance $\operatorname{dist}_{L^{2}(W)}\left\{z^{-1} e, \operatorname{span}\left\{z^{n} \mathbb{C}^{d}: n \geq 0\right\}\right\}=\operatorname{dist}_{L^{2}(W)}\left\{e, \operatorname{span}\left\{z^{n} \mathbb{C}^{d}: n>0\right\}\right\}$.

By the vectorial version of the Szegö theorem (see [7]) this distance is exactly $\|F(0) e\|$. Using the Möbius transformation of the disk one can get from there

$$
\operatorname{dist}_{L^{2}(W)}\left\{\frac{\left(1-|\lambda|^{2}\right)^{1 / 2}}{z-\lambda} e, \operatorname{span}\left\{z^{n} \mathbb{C}^{d}: n \geq 0\right\}\right\}=\|F(\lambda) e\|_{\mathbb{C}^{d}} .
$$

Writing the Fourier series expansion of $\frac{\left(1-|\lambda|^{2}\right)^{1 / 2}}{z-\lambda}$

$$
\frac{\left(1-|\lambda|^{2}\right)^{1 / 2}}{z-\lambda}=\left(1-|\lambda|^{2}\right)^{1 / 2} \sum_{n=0}^{\infty} \lambda^{n} z^{-(n+1)}
$$

one can see that for any fixed $N>0$ the function $\frac{\left(1-|\lambda|^{2}\right)^{1 / 2}}{z-\lambda} e$ is almost in the "past" $X_{-N}$ as $|\lambda| \rightarrow 1$. Namely,

$$
\begin{aligned}
f_{\lambda} & =\frac{\left(1-|\lambda|^{2}\right)^{1 / 2}}{z-\lambda} e \\
& =\left(1-|\lambda|^{2}\right)^{1 / 2} \sum_{n=0}^{N-1} \lambda^{n} z^{-(n+1)} e+\left(1-|\lambda|^{2}\right)^{1 / 2} \sum_{n=N}^{\infty} \lambda^{n} z^{-(n+1)} e \\
& =f_{\lambda}^{1}+f_{\lambda}^{2},
\end{aligned}
$$


where $f_{\lambda}^{2} \in X_{-N}$, and $f_{\lambda}^{1}$ is small,

$$
\begin{aligned}
\frac{\left\|f_{\lambda}^{1}\right\|_{L^{2}(W)}}{\left\|f_{\lambda}\right\|_{L^{2}(W)}} & \leq \frac{\left(1-|\lambda|^{2}\right)^{1 / 2} N \cdot\|e\|_{L^{2}(W)}}{(W(\lambda) e, e)_{\mathbb{C}^{d}}^{1 / 2}} \\
& =\frac{\left(1-|\lambda|^{2}\right)^{1 / 2} N \cdot(W(0) e, e)_{\mathbb{C}^{d}}^{1 / 2}}{(W(\lambda) e, e)_{\mathbb{C}^{d}}^{1 / 2}} \leq N \alpha(|\lambda|)^{1 / 2} \rightarrow 0,
\end{aligned}
$$

as $|\lambda| \rightarrow 1$, where $\alpha($.$) is as in Lemma 4.2$ and Corollary 4.3.

Since $X_{0}$ and $X^{N}$ are asymptotically orthogonal, the shift invariance implies that the subspaces $X_{-N}$ and $X^{0}$ are asymptotically orthogonal as well. Taking $|\lambda| \rightarrow 1$ and then $N \rightarrow \infty$ we can conclude that

$$
\begin{aligned}
& \|F(\lambda) e\|_{\mathbb{C}^{d}} /\left\|W(\lambda)^{1 / 2} e\right\|_{\mathbb{C}^{d}} \\
& =\operatorname{dist}_{L^{2}(W)}\left\{\frac{\left(1-|\lambda|^{2}\right)^{1 / 2}}{z-\lambda} e, \operatorname{span}\left\{z^{n} \mathbb{C}^{d}: n \geq 0\right\}\right\} /\left\|f_{\lambda}\right\|_{L^{2}(W)} \\
& \geq 1-\beta(|\lambda|) \rightarrow 1,
\end{aligned}
$$

where $\beta($.$) depends only on the Muckenhoupt norm of W$ and $\beta(|\lambda|) \rightarrow 0$ as $|\lambda| \rightarrow 1$.

The last inequality implies

$$
\left\|W(\lambda)^{1 / 2} F(\lambda)^{-1}\right\| \leq(1-\beta(|\lambda|))^{-1} .
$$

Note that since $\|F(\lambda) e\|_{\mathbb{C}^{d}} /\left\|W(\lambda)^{1 / 2} e\right\|_{\mathbb{C}^{d}} \leq 1$ for all $e \in \mathbb{C}^{d}$, we have

$$
\left\|W(\lambda)^{1 / 2} F(\lambda)^{-1}\right\| \geq 1 \text {. }
$$

We will show a little later that under assumptions of the theorem the subspaces $X_{0}$ and $X^{N}$ in the weighted space $L^{2}\left(W^{-1}\right)$ are asymptotically orthogonal as well. The factorization $W=F^{*} F$ yields the factorization $W^{-1}=F^{-1}\left(F^{-1}\right)^{*}$ of $W^{-1}$. Similarly to the previous case

$$
\begin{aligned}
& \text { dist }_{L^{2}\left(W^{-1}\right)}\left\{\frac{\left(1-|\lambda|^{2}\right)^{1 / 2}}{1-\bar{\lambda} z} e, \operatorname{span}\left\{z^{n} \mathbb{C}^{d}: n \geq 0\right\}\right\} \\
& =\left\|F^{-1}(\lambda)^{*} e\right\|_{\mathbb{C}^{d}}=\left\|F(\lambda)^{-1 *} e\right\|_{\mathbb{C}^{d}} .
\end{aligned}
$$

Acting as before we get

$$
\left\|W^{-1}(\lambda)^{1 / 2} F(\lambda)^{*}\right\| \leq\left(1-\beta_{1}(|\lambda|)\right)^{-1}
$$

where $\beta_{1}(|\lambda|) \rightarrow 0$ as $|\lambda| \rightarrow 1$.

Combining (4.2) and (4.3) we get

$$
\left\|W(\lambda)^{1 / 2} W^{-1}(\lambda)^{1 / 2}\right\| \leq(1-\beta(|\lambda|))^{-1}\left(1-\beta_{1}(|\lambda|)\right)^{-1} \rightarrow 1 \quad \text { as }|\lambda| \rightarrow 1 .
$$

So, we completed the proof modulo the following lemma. 
This lemma also gives us the equivalence $1 \Longleftrightarrow 2$.

Lemma 4.4. Under assumptions of Theorem 4.1 the weight $W^{-1}$ is a spectral density of a completely regular process, i.e., the spaces $X_{0}$ and $X^{N}$ are asymptotically orthogonal (as $N \rightarrow \infty$ ) in the weighted space $L^{2}\left(W^{-1}\right)$.

Proof. It is enough to show that

$$
\left\|P_{+} \mid \operatorname{span}\left\{X_{0}, X^{N}\right\}\right\|_{L^{2}\left(W^{-1}\right) \rightarrow L^{2}\left(W^{-1}\right)} \rightarrow 1 \quad \text { as } N \rightarrow \infty .
$$

The later is true because

$$
\begin{aligned}
& \left\|P_{+} \mid \operatorname{span}\left\{X_{0}, X^{N}\right\}\right\|_{L^{2}\left(W^{-1}\right) \rightarrow L^{2}\left(W^{-1}\right)} \\
& =\left\|W^{-1 / 2}\left(P_{+} \mid \operatorname{span}\left\{X_{0}, X^{N}\right\}\right) W^{1 / 2}\right\|_{L^{2} \rightarrow L^{2}} \\
& =\left\|W^{1 / 2}\left(P_{+} \mid \operatorname{span}\left\{X_{0}, X^{N}\right\}\right) W^{-1 / 2}\right\|_{L^{2} \rightarrow L^{2}} \\
& =\left\|P_{+} \mid \operatorname{span}\left\{X_{0}, X^{N}\right\}\right\|_{L^{2}(W) \rightarrow L^{2}(W)}
\end{aligned}
$$

and

$$
\left\|P_{+} \mid \operatorname{span}\left\{X_{0}, X^{N}\right\}\right\|_{L^{2}(W) \rightarrow L^{2}(W)} \rightarrow 1 \quad \text { as } N \rightarrow \infty
$$

(since $X_{0}$ and $X^{N}$ are asymptotically orthogonal in $L^{2}(W)$ ).

\section{Vanishing Carleson measures.}

Recall that $W(\lambda)$ and $W^{-1}(\lambda)$ denote harmonic extensions at the point $\lambda \in \mathbb{D}$ of the weights $W$ and $W^{-1}$ respectively.

Lemma 5.1. Let a matrix weight $W$ satisfy

$$
\lim _{|\lambda| \rightarrow 1}\left\|W(\lambda)^{1 / 2}\left(W^{-1}\right)(\lambda)^{1 / 2}\right\|=1 .
$$

Then

$$
\limsup _{|\lambda| \rightarrow 1}\{\operatorname{det}(W(\lambda)) \exp (-[\log \operatorname{det} W](\lambda))\}=1 .
$$

Proof. First of all let us notice that the assumption of the lemma implies that $W, W^{-1} \in L^{1}(\mathbb{T})$, therefore $\log (\operatorname{det} W) \in L^{1}(\mathbb{T})$. Therefore there exists a factorization $W=F^{*} F$ a.e. on $\mathbb{T}$, where $F$ is an outer function in $H^{2}\left(M_{d \times d}\right)$.

Since $F$ is an outer function in $H^{2}$, $\operatorname{det} F$ is an outer function in $H^{2 / d}$. Therefore

$$
|\operatorname{det} F(z)|=\exp \{(\log |\operatorname{det} F|)(z)\}=\exp \left\{\frac{1}{2}(\log \operatorname{det} W)(z)\right\} .
$$

It is well known fact that $F^{*}(z) F(z) \leq W(z)$ for any $z \in D$, where $\leq$ means the inequality for quadratic forms. There are many proofs of this fact, for example it admits a very simple operator-theoretic interpretation 
which is in fact hidden in the proof of Theorem 4.1. Explanation that we present here is more function-theoretic: Direct computation shows that

$$
\Delta\left(F(z)^{*} F(z)\right)=4\left(\bar{\partial} F(z)^{*}\right)(\partial F(z))=4(\partial F(z))^{*}(\partial F(z)) \geq 0,
$$

so for any $e \in \mathbb{C}^{d}$ the function $\|F(z) e\|^{2}$ is subharmonic and coincide with $(W(\xi) e, e)$ on $\mathbb{T}$.

We can do the same factorization for $W^{-1}$. Namely, let $G$ be an outer matrix-valued function in $H^{2}\left(M_{d \times d}\right)$ such that $W^{-1}=G^{*} G$ on $\mathbb{T}$. We should point out to the reader that in general $G$ does not necessarily coincide with $F^{-1}$. However, applying (5.1) to $G$ one can conclude that

$$
|\operatorname{det} G(z)|=\exp \left\{\frac{1}{2}\left(\log \operatorname{det} W^{-1}\right)(z)\right\}=|\operatorname{det} F(z)|^{-1} .
$$

Now we are in position to prove the lemma. By the assumption

$$
\lim _{|z| \rightarrow 1}\left\|W(z)^{1 / 2}\left(W^{-1}\right)(z)^{1 / 2}\right\|=1,
$$

and therefore,

$$
\lim _{|z| \rightarrow 1}\left|\operatorname{det}(W(z)) \operatorname{det}\left(\left(W^{-1}\right)(z)\right)\right|=1 .
$$

Using (5.2) one can rewrite the last identity as

$$
\lim _{|z| \rightarrow 1}\left\{\left[\operatorname{det} W(z) /|\operatorname{det} F(z)|^{2}\right]\left[\operatorname{det} W^{-1}(z) /|\operatorname{det} G(z)|^{2}\right]\right\}=1 .
$$

Since $F(z)^{*} F(z) \leq W(z)$ and $G(z)^{*} G(z) \leq W^{-1}(z)$, expressions in brackets are at least 1 , so, taking into account (5.1) we get

$$
\lim _{|z| \rightarrow 1}[\operatorname{det} W(z) / \exp \{(\log \operatorname{det} W)(z)\}]=0
$$

or equivalently

$$
\lim _{|z| \rightarrow 1} \log \{\operatorname{det}(W(z))\}-(\log \operatorname{det} W)(z)=0 .
$$

Theorem 5.2. A matrix weight $W$ satisfies

$$
\limsup _{|\lambda| \rightarrow 1}\{\operatorname{det}(W(\lambda)) \exp (-[\log \operatorname{det} W](\lambda))\}=1
$$

if and only if the measures

$$
\left\|W(z)^{-1 / 2}\left(\frac{\partial}{\partial x} W(z)\right) W(z)^{-1 / 2}\right\|^{2}\left(1-|z|^{2}\right) d x d y
$$

and

$$
\left\|W(z)^{-1 / 2}\left(\frac{\partial}{\partial y} W(z)\right) W(z)^{-1 / 2}\right\|^{2}\left(1-|z|^{2}\right) d x d y
$$

are vanishing Carleson measures. 
The implication $3 \Longrightarrow 4$ of Theorem 1.3 follows immediately from Theorem 5.2 and Lemma 5.1 .

To prove the theorem we need the following well known description of vanishing Carleson measures:

Lemma 5.3. A measure $\mu$ in the unit disk $\mathbb{D}$ is a vanishing Carleson measure if and only if

$$
\lim _{|\lambda| \rightarrow 1} \int_{\mathbb{D}} \frac{1-|\lambda|^{2}}{|1-\bar{\lambda} z|^{2}} d \mu(z)=0 .
$$

We also need the following lemma that was proved in [11], see Lemma 3.1 there.

Lemma 5.4. Let $W$ be a harmonic function of $n$ variables with values in the space of strictly positive $d \times d$ matrices $\left(W(x)=W(x)^{*}>0 \forall x\right)$. Then

$$
\Delta(\log (\operatorname{det} W))=-\sum_{j=1}^{n} \operatorname{trace}\left(\left(W^{-1 / 2} \frac{\partial W}{\partial x_{j}} W^{-1 / 2}\right)^{2}\right) \text {. }
$$

Proof of Theorem 5.2. The proof below follows the lines of the proof of Theorem 3.2 of $[\mathbf{1 1}]$.

By Green's formula and Lemma 5.4

$$
\begin{aligned}
& \log \{\operatorname{det}(W(s))\}-(\log \operatorname{det} W)(s) \\
& =-\frac{1}{2 \pi} \iint_{\mathbb{D}} \log \left|\frac{1-\bar{s} z}{z-s}\right| \Delta \log \{\operatorname{det}(W(z))\} d x d y \\
& =\frac{1}{4 \pi} \iint_{\mathbb{D}}\left\{\operatorname{trace}\left(W(z)^{-1 / 2} \frac{\partial W(z)}{\partial x} W(z)^{-1 / 2}\right)^{2}\right. \\
& \left.\quad+\operatorname{trace}\left(W(z)^{-1 / 2} \frac{\partial W(z)}{\partial y} W(z)^{-1 / 2}\right)^{2}\right\} \log \left|\frac{1-\bar{s} z}{z-s}\right|^{2} d x d y .
\end{aligned}
$$

Using an elementary inequality $\log (1 / a) \geq 1-a$ for $0<a \leq 1$ and the fact that $\|A\| \leq \operatorname{trace} A$ for a nonnegative matrix $A$, the last integral is at least

$$
\begin{aligned}
& \frac{1}{4 \pi} \iint_{\mathbb{D}}\left\|W(z)^{-1 / 2} \frac{\partial W(z)}{\partial x} W(z)^{-1 / 2}\right\|^{2} \log \left|\frac{1-\bar{s} z}{z-s}\right|^{2} d x d y \\
& \geq \frac{1}{4 \pi} \iint_{\mathbb{D}}\left\|W(z)^{-1 / 2} \frac{\partial W(z)}{\partial x} W(z)^{-1 / 2}\right\|^{2}\left(1-\left|\frac{z-s}{1-\bar{s} z}\right|^{2}\right) d x d y \\
& =\iint_{\mathbb{D}}\left\|W(z)^{-1 / 2} \frac{\partial W(z)}{\partial x} W(z)^{-1 / 2}\right\|^{2} \cdot \frac{\left(1-|s|^{2}\right)\left(1-|z|^{2}\right)}{|1-\bar{s} z|^{2}} d x d y .
\end{aligned}
$$


Together with (5.4) this imply

$$
\lim _{|s| \rightarrow 1} \iint_{\mathbb{D}} \frac{\left(1-|s|^{2}\right)}{|1-\bar{s} z|^{2}} \cdot\left\|W(z)^{-1 / 2} \frac{\partial W(z)}{\partial x} W(z)^{-1 / 2}\right\|^{2}\left(1-|z|^{2}\right) d x d y=0
$$

that yields that the measure $\left\|W(z)^{-1 / 2}\left(\frac{\partial}{\partial x} W(z)\right) W(z)^{-1 / 2}\right\|^{2}\left(1-|z|^{2}\right) d x d y$ is a vanishing Carleson measure.

The measure $\left\|W(z)^{-1 / 2}\left(\frac{\partial}{\partial y} W(z)\right) W(z)^{-1 / 2}\right\|^{2}\left(1-|z|^{2}\right) d x d y$ is treated similarly.

To prove the opposite implication, let us estimate the integral

$$
\iint_{\mathbb{D}} \operatorname{trace}\left(W(z)^{-1 / 2} \frac{\partial W(z)}{\partial x} W(z)^{-1 / 2}\right)^{2} \log \left|\frac{1-\bar{s} z}{z-s}\right|^{2} d x d y
$$

(the integral with $\partial W / \partial y$ can be estimated absolutely the same way). Denote by $b_{s}$ a Blaschke factor with zero at the point $s, b_{s}(z)=(z-s)(1-\bar{s} z)^{-1}$.

First of all, we can estimate the trace by $d \cdot\|\cdot\|$, where $d$ is dimension of the space. So we can estimate the integral by

$$
\begin{aligned}
& C \iint_{\mathbb{D}}\left\|W(z)^{-1 / 2} \frac{\partial W(z)}{\partial x} W(z)^{-1 / 2}\right\|^{2} \log \left|b_{s}(z)\right|^{-2} d x d y \\
& =\iint_{\left|b_{s}(z)\right|<\varepsilon} \cdots+\iint_{\left|b_{s}(z)\right| \geq \varepsilon} \cdots .
\end{aligned}
$$

To estimate the second integral we notice that

$$
\log \left|b_{s}(z)\right|^{-2} d x d y \leq C(\varepsilon) \frac{\left(1-|s|^{2}\right)\left(1-|z|^{2}\right)}{|1-\bar{s} z|^{2}}
$$

for $\left|b_{s}(z)\right| \geq \varepsilon$, and since the measure is a vanishing Carleson measure we can make the integral as small as we want when $|s| \rightarrow 1$.

To estimate the first integral let make a trivial observation: If $w \in L^{1}(\mathbb{T})$, $w \geq 0$ and $w(z)$ denotes its harmonic extension at the point $z$, then for all $z$ such that $|z| \leq 1 / 2$ (and therefore for all $z$ such that $|z|<\varepsilon \leq 1 / 2$ )

$$
\frac{\partial}{\partial x} w(z) \leq C w(0)
$$

where $C$ is an absolute constant. Combining this observation with the Harnack inequality $w(0) \leq C^{\prime} w(z),|z| \leq 1 / 2$, and applying it to functions $w()=.(W(\cdot) e, e)_{\mathbb{C}^{d}}$ we get the inequality for quadratic forms

$$
\frac{\partial}{\partial x} W(z) \leq C \varepsilon W(0) \leq C_{1} W(z) .
$$

This implies

$$
\left\|W(z)^{-1 / 2}\left(\frac{\partial}{\partial x} W(z)\right) W(z)^{-1 / 2}\right\| \leq C_{1}, \quad \forall z:|z|<\varepsilon \leq 1 / 2 .
$$


Using the Möbius transformation $z \mapsto b_{s}(z)$ we get

$$
\left\|W(z)^{-1 / 2}\left(\frac{\partial}{\partial x} W(z)\right) W(z)^{-1 / 2}\right\| \leq C \varepsilon, \quad \forall z:\left|b_{s}(z)\right|<\varepsilon \leq 1 / 2 .
$$

Since

$$
\iint_{\left|b_{s}(z)\right| \leq \varepsilon} \log \left|b_{s}(z)\right|^{-2} d x d y \leq C \varepsilon^{2} \log \frac{1}{\varepsilon},
$$

we can estimate the first integral by $C \varepsilon^{2} \log (1 / \varepsilon)$; we can make this number as small as we want by picking sufficiently small $\varepsilon$.

\section{Embedding theorem and equivalent norms.}

By analogy with the scalar case (see [12]) we will say that a matrix weight $W$ satisfies the invariant $A_{\infty}$ condition if

$\left(\operatorname{inv} A_{\infty}\right)$

$$
\sup _{s \in \mathbb{D}}\{\operatorname{det}(W(s)) \exp (-[\log \operatorname{det} W](s))\}<\infty .
$$

The supremum is called the invariant $A_{\infty}$ norm of $W$.

Theorem 5.2 implies that if the measures

$$
\left\|W(z)^{-1 / 2}\left(\frac{\partial}{\partial x} W(z)\right) W(z)^{-1 / 2}\right\|^{2}\left(1-|z|^{2}\right) d x d y
$$

and

$$
\left\|W(z)^{-1 / 2}\left(\frac{\partial}{\partial y} W(z)\right) W(z)^{-1 / 2}\right\|^{2}\left(1-|z|^{2}\right) d x d y
$$

are vanishing Carleson measures then the weight $W$ satisfies the invariant $A_{\infty}$ condition.

Literally repeating the proof of Theorem 5.2 one can obtain that the weight $W$ satisfies the invariant $A_{\infty}$ condition if and only if the above measures are Carleson.

We will need the following "embedding theorem". More general result was proved in [11], Lemma 4.1.

Lemma 6.1. Let $W$ be a matrix weight satisfying the invariant $A_{\infty}$ condition, and let $\mu$ be a Carleson measure with the Carleson norm $\|\mu\|_{C}$. Then for any analytic (or antianalytic) vector-function $f$, the following inequality holds,

$$
\iint_{\mathbb{D}}(W(z) f(z), f(z)) d \mu(z) \leq C\|\mu\|_{C} \int_{\mathbb{T}}(W(\xi) f(\xi), f(\xi)) d m(\xi),
$$

where the constant $C$ depends the dimension $d$ and the invariant $A_{\infty}$ norm of $W$. 
Proof. The invariant $A_{\infty}$ condition implies that $\log \operatorname{det} W \in L^{1}$, so there exists (see [7]) an outer function $F \in H^{2}\left(M_{d \times d}\right)$ such that $W=F^{*} F$. It is well known (see again [7]) that

$$
|\operatorname{det} F(z)|=\exp \left\{\frac{1}{2}[\log \operatorname{det} W](z)\right\} .
$$

It is well known and it was already shown it in the proof of Lemma 5.1 that $F(z)^{*} F(z) \leq W(z)$. Hence

$$
\left\|W(z)^{1 / 2} F(z)^{-1} e\right\| \geq\|e\|, \quad e \in \mathbb{C}^{d} .
$$

Since

$$
\left|\operatorname{det}\left\{W(z)^{1 / 2} F(z)^{-1}\right\}\right|=\{\operatorname{det}(W(\lambda)) \exp (-[\log \operatorname{det} W](\lambda))\}^{1 / 2} \leq C
$$

we can estimate

$$
\left\|W(z)^{1 / 2} F(z)^{-1} e\right\| \leq C .
$$

Together with (6.5) it implies that $(W(z) e, e)$ and $\|F(z) e\|^{2}$ are equivalent in a sense of two-sided estimate. Therefore

$$
\begin{aligned}
& \iint_{\mathbb{D}}(W(z) f(z), f(z)) d \mu(z) \\
& \leq C \iint_{\mathbb{D}}(F(z) f(z), F(z) f(z)) d \mu(z) \\
& \leq C\|\mu\|_{C} \int_{\mathbb{T}}(F(\xi) f(\xi), F(\xi) f(\xi)) d m(\xi) \\
& =C\|\mu\|_{C} \int_{\mathbb{T}}(W(\xi) f(\xi), f(\xi)) d m(\xi) .
\end{aligned}
$$

We also need the following simple lemma.

Lemma 6.2 (equivalence of weighted norms). Let $W$ be a matrix weight satisfying the invariant $A_{\infty}$ condition. There exist a constant $C$ such that for any analytic or antianalytic vector-function $f$ in $L^{2}(W)$ satisfying $f(0)=0$

$$
\frac{1}{C} \int_{\mathbb{T}}(W f, f) d m \leq \iint_{\mathbb{D}}\left(W(z) f^{\prime}(z), f^{\prime}(z)\right) \log \frac{1}{|z|} d x d y \leq C \int_{\mathbb{T}}(W f, f) d m .
$$

Proof. Let us recall the the operators $\partial$ and $\bar{\partial}$ are defined as

$$
\partial f=\frac{1}{2}\left(\frac{\partial f}{\partial x}-i \frac{\partial f}{\partial y}\right), \quad \bar{\partial} f=\frac{1}{2}\left(\frac{\partial f}{\partial x}+i \frac{\partial f}{\partial y}\right) .
$$

Recall that for analytic functions $\partial f=f^{\prime}$ and $\bar{\partial} f=0$. 
Let $f$ be an analytic function, $f(0)=0$. Using the Green's formula and taking into account that $f(0)=0$ and $\Delta=4 \partial \bar{\partial}=4 \bar{\partial} \partial$ we get

$$
\begin{aligned}
\int_{\mathbb{T}}(W f, f) d m= & \frac{1}{2 \pi} \iint_{\mathbb{D}} \Delta(W(z) f(z), f(z)) \log \frac{1}{|z|} d x d y \\
= & \frac{2}{\pi} \iint_{\mathbb{D}}\left(\bar{\partial} W(z) f^{\prime}(z), f(z)\right) \log \frac{1}{|z|} d x d y \\
& +\frac{2}{\pi} \iint_{\mathbb{D}}\left(\partial W(z) f(z), f^{\prime}(z)\right) \log \frac{1}{|z|} d x d y \\
& +\frac{2}{\pi} \iint_{\mathbb{D}}\left(W(z) f^{\prime}(z), f^{\prime}(z)\right) \log \frac{1}{|z|} d x d y \\
= & \frac{2}{\pi}\left(\mathcal{I}_{1}+\mathcal{I}_{2}+\mathcal{I}_{3}\right) .
\end{aligned}
$$

The last integral $\mathcal{I}_{3}$ is exactly the integral we want to estimate. Let us denote $A^{2}:=\int_{\mathbb{T}}(W f, f) d m, B^{2}:=\mathcal{I}_{3}$. We want to show that $A \asymp B$ in a sense of two sided estimate. Let us estimate $\mathcal{I}_{1}$ :

$\left|\mathcal{I}_{1}\right|$

$$
\begin{gathered}
=\mid \iint_{\mathbb{D}}\left(W(z)^{-1 / 2} \bar{\partial} W(z) W(z)^{-1 / 2} W(z)^{1 / 2} f^{\prime}(z), W(z)^{1 / 2} f(z)\right) \\
\cdot \log \frac{1}{|z|} d x d y \mid \\
\leq \mid \iint_{\mathbb{D}}\left\|W(z)^{-1 / 2} \bar{\partial} W(z) W(z)^{-1 / 2}\right\| \cdot\left\|W(z)^{1 / 2} f^{\prime}(z)\right\| \cdot\left\|W(z)^{1 / 2} f(z)\right\| \\
\cdot \log \frac{1}{|z|} d x d y \mid
\end{gathered}
$$$$
\leq\left(\iint_{\mathbb{D}}\left\|W(z)^{-1 / 2} \bar{\partial} W(z) W(z)^{-1 / 2}\right\|^{2}(W(z) f(z), f(z))_{\mathbb{C}^{d}} \log \frac{1}{|z|} d x d y\right)^{1 / 2}
$$$$
\cdot\left(\iint_{\mathbb{D}}\left(W(z) f^{\prime}(z), f^{\prime}(z)\right)_{\mathbb{C}^{d}} \log \frac{1}{|z|} d x d y\right)^{1 / 2} .
$$

The measure $\left\|W(z)^{-1 / 2} \bar{\partial} W(z) W(z)^{-1 / 2}\right\|^{2} \log \frac{1}{|z|} d x d y$ is Carleson, so by Lemma 6.1 the first term in the product is estimated by $K A$ ( $K$ is a constant). The second term is just $B$ so $\left|\mathcal{I}_{1}\right| \leq K A B$. Similarly $\left|\mathcal{I}_{2}\right| \leq K A B$. So

$$
A^{2}=B^{2}+\mathcal{I}_{1}+\mathcal{I}_{2}
$$

where

$$
\left|\mathcal{I}_{1}\right|,\left|\mathcal{I}_{2}\right| \leq K A B
$$

This immediately implies

$$
\frac{1}{C} A \leq B \leq C A
$$


for an appropriate choice of $C$.

\section{Proof of the implication $6 \Longrightarrow 1$.}

To prove the implication $6 \Longrightarrow 1$ we need to estimate $\int_{\mathbb{T}}\left(W z^{n} f, g\right) d m, f \in$ $X^{0}, g \in X_{0},\|f\|_{L^{2}(W)}=\|g\|_{L^{2}(W)}=1$.

Using the Green's formula and taking into account that $g(0)=0$ and $\Delta=4 \partial \bar{\partial}=4 \bar{\partial} \partial$ we get

$$
\begin{aligned}
\int_{\mathbb{T}}\left(W z^{n} f, g\right) d m= & \frac{1}{2 \pi} \iint_{\mathbb{D}} \Delta\left(W(z) z^{n} f(z), g(z)\right)_{\mathbb{C}^{d}} \log \frac{1}{|z|} d x d y \\
= & \frac{2}{\pi} \iint_{\mathbb{D}}\left(\bar{\partial} W(z) \partial\left(z^{n} f(z)\right), g(z)\right)_{\mathbb{C}^{d}} \log \frac{1}{|z|} d x d y \\
& +\frac{2}{\pi} \iint_{\mathbb{D}}\left(\bar{\partial} W(z)\left(z^{n} f(z)\right), \bar{\partial} g(z)\right)_{\mathbb{C}^{d}} \log \frac{1}{|z|} d x d y \\
= & \frac{2}{\pi}\left(\mathcal{I}_{1}+\mathcal{I}_{2}\right) .
\end{aligned}
$$

The second integral is easy to estimate:

$\left|\mathcal{I}_{2}\right|$

$$
\begin{aligned}
& =\mid \iint_{\mathbb{D}}\left(W(z)^{-1 / 2} \bar{\partial} W(z) W(z)^{-1 / 2} W(z)^{1 / 2}\left(z^{n} f(z)\right), W(z)^{1 / 2} \bar{\partial} g(z)\right)_{\mathbb{C}^{d}} \\
& \cdot \log \frac{1}{|z|} d x d y \mid \\
& \leq \iint_{\mathbb{D}}\left\|W(z)^{-1 / 2} \bar{\partial} W(z) W(z)^{-1 / 2}\right\| \cdot\left\|W(z)^{1 / 2}\left(z^{n} f(z)\right)\right\| \cdot\left\|W(z)^{1 / 2} \bar{\partial} g(z)\right\| \\
& \quad \cdot \log \frac{1}{|z|} d x d y \\
& \leq\left(\iint_{\mathbb{D}}|z|^{2 n} \cdot\left\|W(z)^{-1 / 2} \bar{\partial} W(z) W(z)^{-1 / 2}\right\|^{2} \cdot(W(z) f(z), f(z))_{\mathbb{C}^{d}}\right. \\
& \left.\cdot \cdot \log \frac{1}{|z|} d x d y\right)^{1 / 2} \\
& \quad\left(\iint_{\mathbb{D}}(W(z) \bar{\partial} g(z), \bar{\partial} g(z))_{\mathbb{C}^{d}} \log \frac{1}{|z|} d x d y\right)^{1 / 2} \cdot
\end{aligned}
$$

The last term is equivalent to the norm $\|g\|_{L^{2}(W)}$ (see Lemma 6.2), so by Lemma 6.1

$$
\begin{aligned}
&\left|\mathcal{I}_{2}\right| \leq\|f\|_{L^{2}(W)} \cdot\|g\|_{L^{2}(W)} \\
& \cdot\left\||z|^{2 n} \cdot\right\| W(z)^{-1 / 2} \bar{\partial} W(z) W(z)^{-1 / 2}\left\|\log \frac{1}{|z|} d x d y\right\|_{C}^{1 / 2} .
\end{aligned}
$$


Since the measure $\left\|W(z)^{-1 / 2} \bar{\partial} W(z) W(z)^{-1 / 2}\right\| \log \frac{1}{|z|} d x d y$ is a vanishing Carleson measure, the Carleson norm

$$
\left\||z|^{2 n} \cdot\right\| W(z)^{-1 / 2} \bar{\partial} W(z) W(z)^{-1 / 2}\left\|\log \frac{1}{|z|} d x d y\right\|_{C}^{1 / 2} \rightarrow 0
$$

as $n \rightarrow \infty$. So $\left|\mathcal{I}_{2}\right| \rightarrow 0$ as $n \rightarrow \infty$.

To estimate $\mathcal{I}_{1}$ we pick $r<1$ close to 1 and split the integral into two: $\mathcal{I}_{1}=\iint_{r \mathbb{D}} \cdots+\iint_{\mathbb{D} \backslash r \mathbb{D}} \cdots$ Acting as with $\mathcal{I}_{2}$ we can estimate

$$
\begin{aligned}
& \left|\iint_{X} \ldots\right| \\
& \leq\left(\iint_{X} \cdot\left\|W(z)^{-1 / 2} \bar{\partial} W(z) W(z)^{-1 / 2}\right\|^{2} \cdot(W(z) g(z), g(z))_{\mathbb{C}^{d}}\right. \\
& \left.\cdot \log \frac{1}{|z|} d x d y\right)^{1 / 2} \\
& \quad\left(\iint_{X}\left(W(z) \partial\left(z^{n} f(z)\right), \partial\left(z^{n} f(z)\right)\right)_{\mathbb{C}^{d}} \log \frac{1}{|z|} d x d y\right)^{1 / 2},
\end{aligned}
$$

where $X$ is either $r \mathbb{D}$ or $\mathbb{D} \backslash r \mathbb{D}$. Note that both terms are uniformly bounded.

We can say even more. If $X=r \mathbb{D}$ the second term can be made as small as we wish by picking sufficiently large $n$.

Let now $X=\mathbb{D} \backslash r \mathbb{D}$. The measure $\left\|W(z)^{-1 / 2} \bar{\partial} W(z) W(z)^{-1 / 2}\right\| \log \frac{1}{|z|} d x d y$ is a vanishing Carleson measure, so for $r$ sufficiently close to 1 its restriction onto $\mathbb{D} \backslash r \mathbb{D}$ has the Carleson norm as small as we want. So by Lemma 6.1 the first term is as small as we want if $r$ is sufficiently close to 1.

\section{A counterexample to Peller's conjecture.}

In this section we are going to construct a weight $W$, such that $W^{-1} \in L^{1}$, $\log W \in \mathrm{VMO}$, but the corresponding stationary process is not completely regular (i.e., the weight $W$ does not satisfy any of the conditions 1-6 of Theorem 1.3).

Let

$$
W=U^{*}\left(\begin{array}{cc}
1 & 0 \\
0 & \delta(z)
\end{array}\right) U, \quad U=\left(\begin{array}{cc}
\cos \alpha & -\sin \alpha \\
\sin \alpha & \cos \alpha
\end{array}\right) .
$$

Here

$$
\delta\left(e^{i t}\right)=1 / \log (1 /|t|), \quad-1 / 4 \leq t \leq 1 / 4,
$$

and $\delta$ is a continuous function bounded away from 0 and $\infty$ on the rest of the circle, and

$$
\alpha\left(e^{i t}\right)=(t /|t|) \delta\left(e^{i t}\right)^{1 / 4}, \quad-1 / 4 \leq t \leq 1 / 4,
$$

and again $\alpha$ is continuous on the rest of the circle. 
Then

$\log W=U^{*}\left(\begin{array}{cc}0 & 0 \\ 0 & \log \delta\end{array}\right) U=\left(\begin{array}{ll}\sin ^{2} \alpha \log \delta & \sin \alpha \cos \alpha \log \delta \\ \sin \alpha \cos \alpha \log \delta & \cos ^{2} \alpha \log \delta\end{array}\right)$,

and this matrix clearly belongs to VMO: $\log \delta=\log \log 1 /|t|$ (considered only in a neighborhood of 0 ) is a "typical" unbounded function in VMO, so $\cos ^{2} \alpha \log \delta \in \mathrm{VMO}$, and all other entries of the matrix are continuous.

Let us now show that the weight $W$ does not even satisfies the Muckenhoupt condition $\left(A_{2}\right)$. Direct computations show that

$$
W=\left(\begin{array}{cc}
\cos ^{2} \alpha & -\sin \alpha \cos \alpha \\
-\sin \alpha \cos \alpha & \sin ^{2} \alpha
\end{array}\right)+\delta\left(\begin{array}{cc}
\sin ^{2} \alpha & \sin \alpha \cos \alpha \\
\sin \alpha \cos \alpha & \cos ^{2} \alpha
\end{array}\right)
$$

and

$$
W^{-1}=\left(\begin{array}{cc}
\cos ^{2} \alpha & -\sin \alpha \cos \alpha \\
-\sin \alpha \cos \alpha & \sin ^{2} \alpha
\end{array}\right)+\delta^{-1}\left(\begin{array}{cc}
\sin ^{2} \alpha & \sin \alpha \cos \alpha \\
\sin \alpha \cos \alpha & \cos ^{2} \alpha
\end{array}\right) .
$$

If we pick $I$ to be a symmetric arc $\left[e^{-i \varepsilon}, e^{i \varepsilon}\right](\varepsilon>0$ is small), then off-diagonal entries of $W_{I}$ and $\left(W^{-1}\right)_{I}$ equal 0 , and so we can estimate

$$
\begin{aligned}
W_{I} & \geq C\left(\begin{array}{cc}
\cos ^{2} \alpha(\varepsilon) & 0 \\
0 & \sin ^{2} \alpha(\varepsilon)
\end{array}\right), \\
\left(W^{-1}\right)_{I} & \geq C\left(\begin{array}{cc}
\delta(\varepsilon)^{-1} \sin ^{2} \alpha(\varepsilon) & 0 \\
0 & \delta(\varepsilon)^{-1} \cos ^{2} \alpha(\varepsilon)
\end{array}\right) .
\end{aligned}
$$

Therefore

$$
\left\|\left[W_{I}\right]^{1 / 2}\left[\left(W^{-1}\right)_{I}\right]^{1 / 2}\right\| \geq C \delta(\varepsilon)^{-1} \sin \alpha(\varepsilon) \cos \alpha(\varepsilon) \rightarrow \infty \quad \text { as } \quad \varepsilon \rightarrow 0 .
$$

\section{References}

[1] J.B. Garnett, Bounded Analytic Functions, Acad. Press, NY, 1981.

[2] H. Helson and D. Sarason, Past and future, Math. Scand., 21 (1967), 5-16.

[3] I.A. Ibragimov, Completely regular multidimensional stationary processes with discrete time, Proc. Steklov Inst. Math., 111 (1970), 269-301.

[4] P. Masani and N. Wiener, On bivariate stationary processes and the factorization of matrix-valued functions, Theor. Probability Appl., 4 (1959), 300-308.

[5] V.V. Peller, Hankel operators and multivariate stationary processes, Operator theory: Operator algebras and applications, Part 1, (Durham, NH, 1988), 357-371, Proc. Sympos. Pure Math., 51(1), Amer. Math. Soc., Providence, RI, 1990.

[6] V.V. Peller and S.V. Khruschev, Hankel operators, best approximation, and stationary Gaussian processes, Russian Math. Surveys, 37 (1982), 53-124.

[7] M. Rosenblum and J. Rovnyak, Hardy Classes and Operator Theory, (Oxford Mathematical Monographs) Oxford Science Publications, The Clarendon Press, Oxford University Press, New York, 1985.

[8] Yu.A. Rozanov, Stationary Stochastic Processes, Holden-Day, SF, 1967. 
[9] D. Sarason, An addendum to "Past and future", Math. Scand., 30 (1972), 62-64.

[10] S. Treil and A. Volberg, Wavelets and the angle between past and future, Journal of functional analysis, 143(2) (1997), 269-308.

[11] Continuous frame decomposition and a vector Hunt-Muckenhoupt-Wheeden Theorem, Arkiv för Matematik, 35(2) (1997), 363-386.

[12] S. Treil, A. Volberg and D. Zheng, Hilbert transform, Toeplitz operators and Hankel operators, and invariant $A_{\infty}$ weights, to appear in Rev. Mat. Iberoamericana.

[13] N. Wiener and P. Masani, The prediction theory of multivariate stochastic processes, I. The regularity conditions, Acta Math., 98 (1957), 111-150.

[14] _ The prediction theory of multivariate stochastic processes, II. The linear predicator, Acta Math., 99 (1958), 93-137.

Received February 2, 1998. This research was partially supported by the NSF grant DMS 9622936, binational Israeli-USA grant BSF 00030, and research program at MSRI in the Fall of 1997.

Michigan State University

EAST LANSing, Michigan 48824

E-mail address: treil@math.msu.edu

Michigan State University

East Lansing, Michigan 48824

E-mail address: volberg@math.msu.edu 